\title{
Theoretical and experimental demonstrations of a microfiber-based flexural disc accelerometer
}

\author{
G. Y. Chen, ${ }^{1, *}$ X. L. Zhang, ${ }^{1,2}$ G. Brambilla, ${ }^{1}$ and T. P. Newson ${ }^{1}$ \\ ${ }^{1}$ Optoelectronics Research Centre, University of Southampton, Southampton, SO17 1BJ, UK \\ ${ }^{2}$ College of Optoelectronic Science and Engineering, National University of Defense Technology, Changsha 410073, China \\ ${ }^{*}$ Corresponding author: gyc1g09@orc.soton.ac.uk
}

Received July 7, 2011; revised August 17, 2011; accepted August 22, 2011;

posted August 24, 2011 (Doc. ID 150728); published September 14, 2011

\begin{abstract}
The proof-of-concept demonstration of a microfiber-based flexural disc accelerometer is presented. The reduced microfiber size and bending radii give rise to high device compactness and responsivity. A flexural disc accelerometer manufactured from a $10 \mathrm{~mm}$ long microfiber showed a performance of $\sim 2.2 \mathrm{rad} / \mathrm{g}$, with the responsivity expected to increase proportionally with the microfiber length. () 2011 Optical Society of America

OCIS codes: $\quad 060.2370,120.0280,120.3180,280.4788$.
\end{abstract}

Accelerometers play an important part as existing and next-generation tools for inertial navigation, guidance systems, earthquake monitoring, platform stabilization for space applications, vibration monitoring in machinery, vehicles, vessels, and portable electronics such as free-fall sensors. Fiber-optic accelerometers have several inherent advantages over micro-electromechanicalsystems (MEMS) based accelerometers, such as lower temperature dependence, higher responsivity, and immunity to electromagnetic interference [1-3]. To date, optical accelerometers have been reported in many different forms, with the most extensively researched being compliant cylinders/mandrels $[\underline{4}, \underline{5}]$ and central/edgesupported flexural discs [6-9], which are interferometrybased. Other types include hollow/multicore fiber [2,3], microloop resonator [1], weighted reflective diaphragm [10], and fiber Bragg gratings [11]. In-fiber designs are highly compact, but are limited by low responsivity. Although designs based on compliant cylinders/mandrels and weighted reflective diaphragm showed some positive attributes, notably very high sensitivity $\sim 10^{4} \mathrm{rad} / \mathrm{g}$ [4], they also exhibit small operating bandwidth that is typically below $1 \mathrm{kHz}$. Flexural disc designs exhibit poor responsivity in small packages. Previously, as much as $75 \mathrm{~m}$ of SMF-28 has been used in flexural disc designs for the purpose of high responsivity [9]. Still, the flexural disc designs have operating bandwidths higher than most configurations, e.g. a few kHz. For portable applications where weight and size requirements are stringent, flexural discs face a serious design problem.

In this Letter, we present a solution with a microfiberbased, centrally-supported flexural disc accelerometer that has the potential to achieve higher compactness and higher responsivity than conventional flexural discs that use telecom single-mode optical fibers or even bendinsensitive fiber (BIF). The phase-demodulation is performed by a differential technique on the intensity. Microfibers used in sensors and devices have diameters in the region of $1-3 \mu \mathrm{m}$, meaning that small bend radii can be achieved [12] without inducing significant polarization dependent loss and depolarization of light. Therefore, high device compactness is feasible without deterioration in expected performance. A smaller disc size also implies a higher fundamental resonance frequency, thus higher operating bandwidth. Since microfibers have reduced width, longer lengths are possible on a disc of the same size, leading to a larger response to strain and hence higher responsivity.

The operating principle of the flexural disc accelerometer utilizes axial acceleration, which causes extensive strain in one fiber spiral and compressive strain in another, providing a push-pull enhancement and effectively doubles the accelerometer's response while providing common-mode rejection of pressure and temperature variations. Equations (1) and (2) are used to predict the phase $(\phi)$ responsivity to acceleration $(A)$ of a centrally-supported flexural disc [9]. The stress-optic effect of microfibers are assumed to be the same as bulk silica and approximately equal to doped silica fibers [13]. Equation (1) calculates the length change due to the strain $\varepsilon$ (expressed in terms of pressure changes $\Delta P$ due to acceleration) experienced by a ring of microfiber at radial distance $r$ from the disc center. Equation (2) gives the sensor responsivity ( $\mathrm{rad} / \mathrm{g}$ ) by approximating the total length change $\left(\Delta L_{\text {total }}\right)$ as the sum of cascaded microfiber rings, which is dependent on the microfiber diameter $(D)$ :

$$
\begin{aligned}
& \Delta L=\varepsilon(r, a, b, h, E, \nu) \cdot(2 \pi r), \\
& \frac{\Delta \phi}{\Delta A}=2 \times 9.81 \times 6.32 \pi \frac{h \rho n \Delta L_{\text {total }}}{\lambda \Delta P},
\end{aligned}
$$

where $a$ is the disc radius, $b$ is the central support radius, $h$ is the disc thickness, $E$ is the effective Young's modulus, $\nu$ is the Poisson ratio of the disc, $\rho$ is the effective disc density, $n$ is the effective index of the microfiber core, and $\lambda$ is the wavelength of input light. It is assumed that only a single layer of microfiber is wound on each side of the disc.

Microfibers have strong evanescence fields since they confine light through a refractive index contrast between the silica core and its surrounding. Therefore, adjacent turns within the spiral must be kept apart to prevent optical coupling that leads to resonance. In practice, a $2 \mu \mathrm{m}$ diameter microfiber should have a minimum separation of $20 \mu \mathrm{m}$ for single-mode operation at $1550 \mathrm{~nm}$. Larger fiber diameters would host multiple modes and smaller 
diameters would result in higher optical loss due to more light propagating outside the microfiber.

It is desirable for the accelerometer to exhibit a linear phase response with frequency such that acceleration can be detected without significant distortion. However, the fundamental resonance frequency of the flexural disc sets an upper limit on the sensor bandwidth. An approximate expression for the fundamental resonance frequency is shown in Eq. (3) [6,9], where $\Lambda^{2}$ is the re-

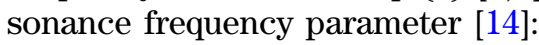

$$
f_{R}=\frac{\Lambda^{2} h}{\pi a^{2}} \sqrt{\frac{E}{12\left(1-v^{2}\right) \rho}} .
$$

Using Eqs. (1) and (2), Fig. 1 compares the simulated responsivity and bandwidth performance (single-layer) of a microfiber with those of a BIF (Corning HI 1060 FLEX) on a carbon-polymer disc. For the microfiber, it is assumed that: $n \approx 1.4, \lambda=1550 \mathrm{~nm}, v=0.42, \rho=$ $1175 \mathrm{~kg} / \mathrm{m}^{3}, b=1.5 \mathrm{~mm}$, and the microfiber is wound from $1.5 \mathrm{~mm}$ to the edge, $D=2 \mu \mathrm{m}$ with separation of $20 \mu \mathrm{m}, h=0.25 \mathrm{~mm}, E=6.1 \mathrm{GPa}, \Lambda^{2}=4.235$. For the $\mathrm{BIF}$, it is assumed that: $n=1.46, \lambda=1550 \mathrm{~nm}, v=$ $0.36, \rho=1515 \mathrm{~kg} / \mathrm{m}^{3}, b=1.5 \mathrm{~mm}$, and the BIF is wound from $5 \mathrm{~mm}$ to the edge, $D=125 \mu \mathrm{m}, h=0.25 \mathrm{~mm}$, $E=79.1 \mathrm{GPa}, \Lambda^{2}=4.235$. The values for density are an estimated average of the composite structure. From the graphs, it is evident that the microfiber leads to much higher responsivity, although the bandwidth is lower. For a disc radius of $12.5 \mathrm{~mm}$, the responsivity is $7958 \mathrm{rad} / \mathrm{g}$ for microfiber compared to $137 \mathrm{rad} / \mathrm{g}$ for BIF. More importantly, the nominal macrobend radius limit of BIF is $5 \mathrm{~mm}$, which sets a lower bound on the disc size. This simulation model is in good agreement with the experimental results reported in [9].

A $2 \mu \mathrm{m}$ diameter, $10 \mathrm{~mm}$ long microfiber was manufactured by tapering a telecom SMF-28 using a ceramic heater [15]. A short length of microfiber is sufficient to demonstrate the principle of the microfiber-based flexural disc accelerometer. The effect of thermal expansion has a great impact on the stability of the accelerometer when incorporated into an interferometer, therefore a carbon-polymer material with a low coefficient of thermal expansion was considered to be suitable for the construction of the disc. In addition, the material has a

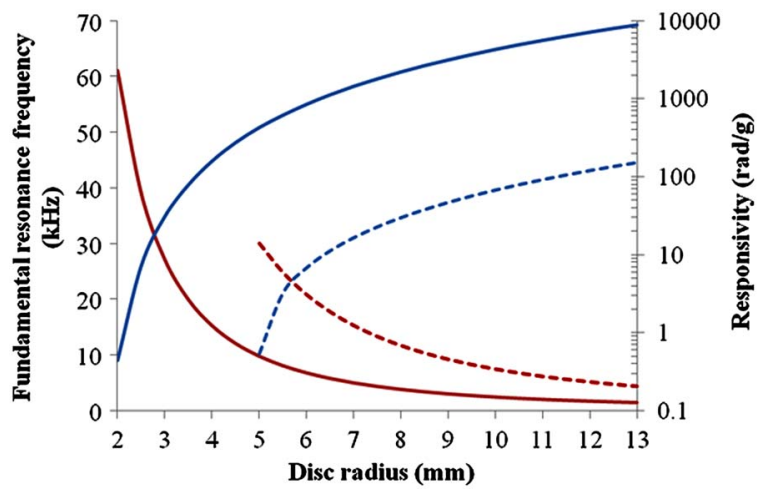

Fig. 1. (Color online) Simulated responsivity (blue) and resonance frequency (red) of microfiber (solid line) and bendinsensitive fiber (dashed line). reasonable density and a low Young's modulus. The disc measures $25 \mathrm{~mm}$ in diameter and $0.5 \mathrm{~mm}$ in thickness. For mounting onto the piezoelectric transducer (PZT) shaker, the central support of the disc consists of a metal screw, $3 \mathrm{~mm}$ in diameter, which was securely fixed to the underside of the disc. The total weight of the disc plus screw was only around $4 \mathrm{~g}$ to prevent overloading the PZT. The microfiber was wound on one side of the disc in a U-shape arrangement, $8 \mathrm{~mm}$ from the disc center. Low-index $(n=1.376)$ U.V. curable polymer (Efiron UV-373) was deposited on the disc before and after the attachment of the microfiber for two purposes: 1) to bind the microfiber and disc together for efficient strain transfer and 2) to provide a uniform effective index to reduce birefringence and optical losses. The amount of polymer was kept to a minimum, as substantial quantities can affect the disc's total mass and flexibility. In addition, drifts in the ambient temperature can cause undesirable thermal expansion and contraction of the polymer that will be transferred to the microfiber, inducing a phase-shift in the interference fringes. The insertion loss of the $10 \mathrm{~mm}$ long microfiber is $0.5 \mathrm{~dB}$.

To test the system, linearly-polarized light at $1550 \mathrm{~nm}$ was launched from a tunable laser source (Agilent 8163B) into a Michelson interferometer with the flexural disc accelerometer connected to a Faraday rotator mirror (FRM) forming the sensing arm and another FRM at the end of the reference arm. The incorporation of FRMs ensures that any change in the polarization state along the two arms will cancel out. The flexural disc was mounted on a PZT shaker (Photon Control), which is controlled by a signal generator (Thurlby Thandar TG210). The returning light was picked up by a photoreceiver (New Focus 1811) and sampled by a digital oscilloscope (Tektronix DPO4054), as illustrated in Fig. 2.

Phase-demodulation was performed during postprocessing of the measurement data. The variation in the light intensity detected at the output of an interferometer can be written as Eq. (4), where $\theta(t)$ is the phasedifference between the two arms of the interferometer. The constants $A$ and $B$ are proportional to the input optical power. $B$, however, also depends on the visibility of the interferometer. When a sinusoidal modulation with frequency $\omega_{0}$ and amplitude $C$ is imposed on the sensing arm of the interferometer, the phase-difference can be expressed by Eq. (5), where $C$ is the phase information of interest and $\phi(t)$ represents the environmental effects. It is assumed that the sinusoidal modulation produces no thermal-induced phase-shift. By performing differentiation on Eq. (5) with respect to time, we obtain Eq. (6) when $\phi(t)$ is equal to $\pi / 2$. The maximum phase-shift due to acceleration is therefore given by Eq. (7), where $B$ can be measured from the interference visibility and $\omega_{0}$ is predetermined by the set frequency:

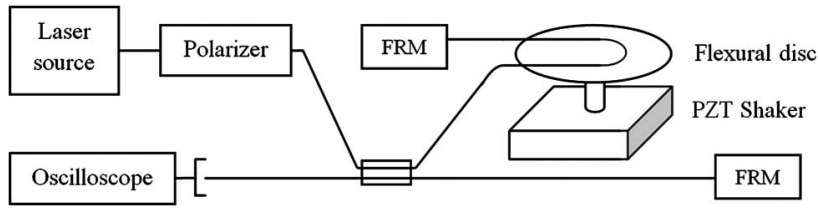

Fig. 2. Schematic diagram of accelerometer system. 


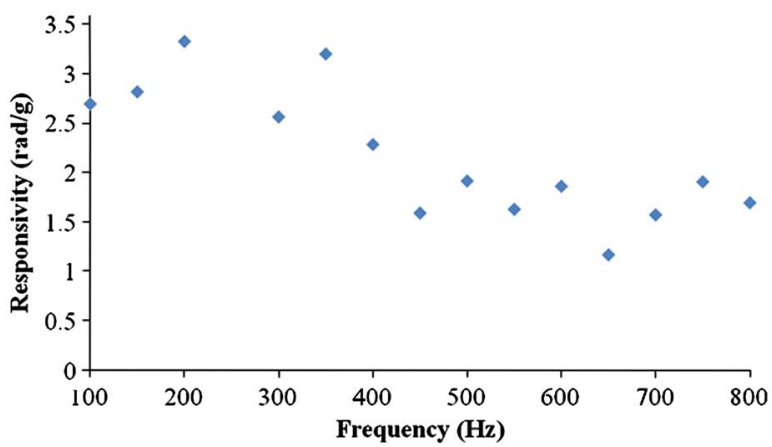

Fig. 3. (Color online) Measured frequency response.

$$
\begin{aligned}
I & =A+B \cos \theta(t) \\
I & =A+B \cos \left[C \cos \left(\omega_{0} t+\Phi\right)+\phi(t)\right], \\
\frac{d I}{d t} & =B C \omega_{0} \sin \left(\omega_{0} t\right) \cos \left[C \cos \left(\omega_{0} t+\Phi\right)\right], \\
C & =\frac{|d I / d t|_{\max }}{B \omega_{0}}
\end{aligned}
$$

A Fabry-Perot etalon was used to measure the acceleration of the PZT shaker. It was constructed by aligning the end of a flat-cleaved fiber suspended perpendicularly to a small, light weight mirror fixed at the center of the disc, mounted on the PZT shaker. The maximum displacement of the PZT shaker ( $s$ ) for a given frequency is calculated from the difference in cavity length between the neutral and peak of oscillation.

$$
s=\frac{N \lambda}{2}
$$

The fringe count is calculated by accumulating the phase of fringes and using arc-sine and arc-cosine functions on fractional fringes. It is feasible to obtain an accurate value for $N$ with resolvable responses in excess of half of a fringe. Acceleration in is calculated from the second derivative of displacement.

$$
|a|=\omega_{0}^{2} s=2 \pi^{2} N \lambda f_{0}^{2}
$$

The measured responsivity of the accelerometer is shown in Fig. 3, which varies between $1.2-3.3 \mathrm{rad} / \mathrm{g}$ for $100-800 \mathrm{~Hz}$. From simulations, the responsivity for a $10 \mathrm{~mm}$ length of microfiber is predicted to be $2.33 \mathrm{rad} / \mathrm{g}$ and $0.24 \mathrm{rad} / \mathrm{g}$ for BIF. The simulated value for microfiber shows some agreement with the experimental results. The variations of responsivity with frequency could be due to the nonuniform polymer deposition on the flexural disc, causing some parts to flex more than others at certain frequencies. The fundamental resonance frequency of a $25 \mathrm{~mm}$ diameter and $0.5 \mathrm{~mm}$ thick carbon-polymer disc was expected to be $1.6 \mathrm{kHz}$ from simulations. However, it was not possible to confirm this experimentally because the frequency range of testing was limited by the operating bandwidth of the PZT sha$\operatorname{ker}(<1 \mathrm{kHz})$. The signal-to-noise ratio was found to be $35.4 \mathrm{~dB}$ with a bandwidth of $20 \mathrm{kHz}$. This corresponds to a phase-sensitivity of $19.1 \mu \mathrm{rad} / \sqrt{\mathrm{Hz}}$. The noise is predominantly caused by intensity and frequency fluctuations of the laser source. The phase sensitivity is combined with the responsivity of the accelerometer to give the acceleration sensitivity as a function of frequency, which can be as low as $57.8 \mu \mathrm{g}\left(g \approx 9.81 \mathrm{~m} / \mathrm{s}^{2}\right)$.

In conclusion, a microfiber-based flexural disc accelerometer has been proposed and demonstrated. It has the potential to reach higher compactness and responsivity than current designs using SMF-28 or BIF. As a proof-ofconcept, we have demonstrated a responsivity of $\sim 2.2 \mathrm{rad} / \mathrm{g}$ between $100-800 \mathrm{~Hz}$ for a $25 \mathrm{~mm}$ diameter, $0.5 \mathrm{~mm}$ thick carbon-polymer disc with a $10 \mathrm{~mm}$ length of $2 \mu \mathrm{m}$ diameter microfiber. The responsivity should increase proportionally with length.

Future work will focus on using longer lengths of microfiber [16] for higher responsivity, as well as extending the testing bandwidth to match the theoretical resonance frequency. For stable and effective operation in a harsh environment, it is necessary to house the accelerometer in a robust metal case. To further reduce the size and weight of the sensor system, it is possible to integrate the coupler of the interferometer into the housing by fabricating it from microfibers [17].

\section{References}

1. C. Hou, Y. Wu, and X. Zeng, Opt. Eng. 49, 014402 (2010).

2. T. Ke, T. Zhu, Y. Rao, and M. Deng, Microw. Opt. Technol. Lett. 52, 2531 (2010).

3. A. Fender, W. N. MacPherson, R. R. J. Maier, J. S. Barton, D. S. George, R. I. Howden, G. W. Smith, B. J. S. Jones, S. McCulloch, X. F. Chen, R. Suo, L. Zhang, and I. Bennion, IEEE Sens. J. 8, 1292 (2008).

4. D. L. Gardner, T. Hofler, S. R. Baker, R. K. Yarber, and S. L. Garrett, J. Lightwave Technol. 5, 953 (1987).

5. R. D. Pechstedt and D. A. Jackson, Appl. Opt. 34, 3009 (1995).

6. D. A. Brown and S. L. Garrett, Proc. SPIE 1367, 282 (1991).

7. Y. Wang, H. Xiao, S. Zhang, F. Li, and Y. Liu, Meas. Sci. Technol. 18, 1763 (2007).

8. Y. Shindo, N. Tsuchida, K. Dobashi, and H. Kamata, in 12th International Conference on Optical Fiber Sensors, Vol. 16 of OSA Technical Digest Series (1997), p. 202.

9. G. A. Cranch and P. J. Nash, J. Lightwave Technol. 18, 1233 (2000).

10. G. A. Gerges, T. P. Newson, and D. A. Jackson, Opt. Lett. 14, 1155 (1989).

11. M. D. Todd, G. A. Johnson, B. A. Althouse, and S. T. Vohra, IEEE Photonic Technol. Lett. 10, 1605 (1998).

12. H. Yu, S. Wang, J. Fu, M. Qiu, Y. Li, F. Gu, and L. Tong, Appl. Opt. 48, 4365 (2009).

13. A. J. Barlow and D. N. Payne, IEEE J. Quantum Electron. 19, 834 (1983).

14. A. W. Leissa, NASA Tech. Doc. Sp-160-N70-18461, 1969.

15. G. Brambilla, J. Opt. 12, 043001 (2010).

16. N. Vukovic, N. G. R. Broderick, M. Petrovich, and G. Brambilla, IEEE Photonic Technol. Lett. 20, 1264 (2008).

17. Y. Li and L. Tong, Opt. Lett. 33, 303 (2008). 